Portland State University

PDXScholar

Electrical and Computer Engineering Faculty

Publications and Presentations

6-28-2007

\title{
Nature-Inspired Interconnects for Self-Assembled Large-Scale Network-on-Chip Designs
}

Christof Teuscher

Portland State University

Follow this and additional works at: https://pdxscholar.library.pdx.edu/ece_fac

Part of the Electrical and Computer Engineering Commons

Let us know how access to this document benefits you.

\section{Citation Details}

C. Teuscher. Nature-Inspired Interconnects for Self-Assembled Large-Scale Network-on-Chip Designs. Chaos, 17(2):026106, 2007.

This Article is brought to you for free and open access. It has been accepted for inclusion in Electrical and Computer Engineering Faculty Publications and Presentations by an authorized administrator of PDXScholar. Please contact us if we can make this document more accessible: pdxscholar@pdx.edu. 


\title{
Nature-inspired interconnects for self-assembled large-scale network-on-chip designs
}

\author{
Christof Teuscher ${ }^{\mathrm{a})}$ \\ Los Alamos National Laboratory CCS-3, MS-B256, Los Alamos, New Mexico 87545
}

(Received 1 January 2007; accepted 23 April 2007; published online 28 June 2007)

\begin{abstract}
Future nanoscale electronics built up from an Avogadro number of components need efficient, highly scalable, and robust means of communication in order to be competitive with traditional silicon approaches. In recent years, the networks-on-chip (NoC) paradigm emerged as a promising solution to interconnect challenges in silicon-based electronics. Current NoC architectures are either highly regular or fully customized, both of which represent implausible assumptions for emerging bottom-up self-assembled molecular electronics that are generally assumed to have a high degree of irregularity and imperfection. Here, we pragmatically and experimentally investigate important design tradeoffs and properties of an irregular, abstract, yet physically plausible three-dimensional (3D) small-world interconnect fabric that is inspired by modern network-on-chip paradigms. We vary the framework's key parameters, such as the connectivity, number of switch nodes, and distribution of long- versus short-range connections, and measure the network's relevant communication characteristics. We further explore the robustness against link failures and the ability and efficiency to solve a simple toy problem, the synchronization task. The results confirm that (1) computation in irregular assemblies is a promising and disruptive computing paradigm for selfassembled nanoscale electronics and (2) that 3D small-world interconnect fabrics with a power-law decaying distribution of shortcut lengths are physically plausible and have major advantages over local two-dimensional and 3D regular topologies. (C) 2007 American Institute of Physics.
\end{abstract}

[DOI: $10.1063 / 1.2740566]$

It is generally expected that without disruptive new technologies, the ever-increasing computing performance and storage capacity achieved with existing technologies will eventually reach a plateau. In recent years, the importance of interconnects on electronic chips has outrun the importance of transistors as a dominant factor of performance and it is widely conceded that technology alone cannot solve the on-chip global interconnect problem with current design methodologies. Radically new approaches are thus necessary, which also take into account future fabrication technologies, such as, for example, selfassembled molecular electronics. Here, we experimentally explore the properties of nature-inspired interconnects for future self-assembled large-scale on-chip interconnect fabrics. We use an abstract, yet realistic framework inspired by modern network-on-chip paradigms and show that irregularly assembled small-world networks with a power-law decaying distribution of shortcut lengths are physically plausible and have major advantages in terms of performance and robustness over both locally and regularly connected two- and three-dimensional interconnect topologies.

\section{INTRODUCTION}

It is generally expected that without disruptive new technologies, the ever-increasing computing performance (commonly known as Moore's law ${ }^{1}$ ) and the storage capacity

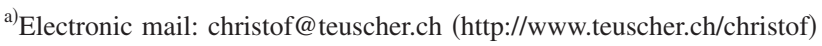

achieved with existing technologies will eventually reach a plateau. ${ }^{2}$ However, there is a lack of consensus on what type of technology and computing architecture holds most promises to keep up the current pace of progress. Among the most contemplated future and emerging technologies are quantum computers, molecular electronics, nanoelectronics, optical computers, and quantum-dot cellular automata (QCA). In this paper, we will primarily focus on self-assembled nanoscale electronics based on nanowires or nanotubes because these fabrication technologies have become quite mature on the physical and device levels. It is, however, still unclear how to develop higher-level computational architectures in a reliable way, although a number of promising approaches have been explored in detail (e.g., Refs. 3-6). As Chen et al. ${ }^{7}$ state, "[i]n order to realize functional nano-electronic circuits, researchers need to solve three problems: invent a nanoscale device that switches an electric current on and off; build a nanoscale circuit that controllably links very large numbers of these devices with each other and with external systems in order to perform memory and/or logic functions; and design an architecture that allows the circuits to communicate with other systems and operate independently on their lower-level details."

Building a scalable computing architecture on top of a potentially very unreliable physical substrate, such as, for example, molecular electronics, is a challenging task, which is guided by a number of major tradeoffs in the design space, ${ }^{8}$ such as the number and the characteristics of the resources available, the required performance, the energy 
consumption, and the reliability. The lack of systematic understanding of these issues and of clear design methodologies makes the process still more an art than a scientific endeavor, and the appearance of novel and nonstandard physical computing devices (e.g., Ref. 6) generally only aggravates these difficulties.

In recent years, the importance of interconnects on electronic chips has outrun the importance of transistors as a dominant factor of performance. ${ }^{9-11}$ The reasons are twofold: (1) The transistor switching speed for traditional silicon is much faster than the average wire delays and (2) the required chip area for interconnects has dramatically increased. The International Technology Roadmap for Semiconductors $(\text { ITRS })^{12}$ lists a number of critical challenges for interconnects and states that, "[i]t is now widely conceded that technology alone cannot solve the on-chip global interconnect problem with current design methodologies." The major challenges are related to delays of nonscalable global interconnects and reliability in general, which lead to the observation that simple scaling will no longer satisfy performance requirements as feature sizes continue to shrink. ${ }^{10}$

In this paper, we experimentally and pragmatically investigate a certain class of irregular and physically plausible three-dimensional (3D) interconnect fabrics, which are likely to be easily and cheaply implementable by selfassembling either nanowires or nanotubes. We will vary the framework's key parameters, such as the connectivity, the number of switch nodes, and the distribution of long- versus short-range connections, and measure the network's relevant communication characteristics and the robustness against failures. Further, we will compare its performance with regular and nearest-neighbor connected two-dimensional (2D) and 3D cellular-automata-like interconnect fabrics. We will also evaluate and compare the performance of a simple task that is frequently used in the cellular automata (CA) community, the synchronization task.

The motivation for investigating alternative and more biologically inspired interconnects that can be selfassembled easily and cheaply are summarized by the following observations:

- Long-range and global connections are costly (in terms of wire delay and of the chip area used) and limit system performance; ${ }^{10}$

- It is unclear whether a precisely regular and homogeneous arrangement of components is needed and possible on a multibillion component or even Avogadro-scale assembly of nanoscale components; ${ }^{6}$

- "[s]elf-assembly makes it relatively easy to form a random array of wires with randomly attached switches;", 13

- Building a perfect system is very hard and expensive.

By using an abstract, yet physically plausible and fabrication-friendly nanoscale computing framework, we show that self-assembled interconnect fabrics with smallworld $(\mathrm{SW})^{14}$ properties have major advantages in terms of performance and robustness over purely regular and nearestneighbor connected fabrics, such as cellular-automata-like topologies (sometimes called NEWS communication, standing for north, east, west, south). While there is ample evi- dence of the superior communication characteristics of small-world and power-law, over locally connected, topologies (see also Sec. II), most abstract models are not physically plausible and are thus of limited significance for realworld implementations. For example, it is very unrealistic to assume a uniform rewiring probability (as in the original Watts-Strogatz model ${ }^{14}$ ) over all possible nodes. Spatial aspects of small-world topologies and wiring-cost perspectives have only recently gained more attention. ${ }^{15-19}$ We call such interconnect topologies nature or bioinspired, because they are physically plausible and similar network topologies are widespread in natural systems.

The goal of this paper is to experimentally investigate important design tradeoffs of self-assembled interconnect fabrics for emerging nanoscale electronics. The main contribution consists of a pragmatic comparison of regular (both 2D and 3D) versus irregular small-world topologies of physically plausible self-assembled network-on-chip (NoC) interconnect fabrics that are inspired by natural networks. We believe that the results will help to make important design decisions for future bottom-up self-assembled computing architectures. The question of how much interconnect we need and how one can efficiently build —or rather self-assembleit, is a very important question, not only for chip design and future molecular electronics in particular, but also for any massively parallel computing architecture in general.

The remainder of the paper is as follows: Section II provides a brief introduction to complex networks and modern network-on-chip paradigms. Section III describes the framework that we use, such as the topologies used, the wire and node models, and how physically plausible the approach is. Section IV reports on five simple experiments that illustrate the main findings, while Sec. V finally concludes the paper.

\section{NETWORKS AND NETWORKS ON CHIP}

\section{A. Complex networks and wiring costs}

Most real networks, such as brain networks, ${ }^{20,21}$ electronic circuits, ${ }^{22}$ the Internet, and social networks share the so-called small-world property. ${ }^{14}$ Compared to purely locally and regularly interconnected networks (such as, for example, the cellular automata interconnect), small-world networks have a very short average distance between any pair of nodes, which makes them particularly interesting for efficient communication.

The classical Watts-Strogatz small-world network ${ }^{14}$ is built from a regular lattice with only nearest-neighbor connections. Every link is then rewired with a rewiring probability $p$ to a randomly chosen node. Thus, by varying $p$, one can obtain a fully regular $(p=0)$ and a fully random $(p=1)$ network topology. The rewiring procedure establishes "shortcuts" in the network, which significantly lower the average distance (i.e., the number of edges to traverse) between any pair of nodes. In the original model, the length distribution of the shortcuts is uniform since a node is chosen randomly. If the rewiring of the connections is done proportional to a power law, $l^{-\alpha}$, where $l$ is the wire length, then we obtain a small-world power-law network. The exponent $\alpha$ affects the network's communication characteristics ${ }^{17}$ and 
navigability, ${ }^{18}$ which is better than in the uniformly generated small-world network. One can think of other distanceproportional distributions for the rewiring, such as, for example, a Gaussian distribution, which has been found between certain layers of the rat's neocortical pyramidal neurons. ${ }^{64}$ Studying the connection probabilities and the average number of connections in biological systems, especially in neural systems, can give us important insights on how nearly optimal systems evolved in nature under limited resources and various other physical constraints.

In a real network, it is fair to assume that local connections have a lower cost (in terms of the associated wire delay and the area required) than long-distance connections. Physically realizing small-world networks with uniformly distributed long-distance connections is thus not realistic and distance, i.e., the wiring cost, needs to be taken into account, a perspective that recently gained increasing attention. ${ }^{15,16}$ On the other hand, a network's topology also directly affects how efficient problems can be solved. For example, it has been shown that both small-world ${ }^{23}$ and random ErdösRényi topologies ${ }^{24}$ offer better performance than regular lattices and are easier to evolve to solve the global synchronization and density classification task, two toy problems commonly used in the cellular automata community.

In summary, there is tradeoff between (1) the physical realizability and (2) the communication characteristics for a network topology. A locally and regularly interconnected topology is in general easy to build and only involves minimal wire and area cost, but it offers poor global communication characteristics and scales up poorly with system size. On the other hand, a random Erdös-Rényi topology scales up well and has a very short-average path length, but it is not physically plausible because it involves costly long-distance connections established independently of the Euclidean distance between the nodes.

\section{B. Addressing interconnect challenges by networks on chip}

The topic of interconnect networks for computers and chips is vast and complex. Here, we will give a brief-and certainly incomplete-overview on communications on chips. From a bird's eye view, the main challenge of interconnect fabrics consists of transferring data between two points of the chip with a minimal latency, minimal energy consumption, and maximal reliability. This job can obviously be done in a wide variety of ways. Compared to computerto-computer networks, one has to generally deal with a more restrictive set of resources and with more constraints to consider. The balance between communication and computation is guided by numerous design tradeoffs and is key to performance. For example, a set of fast processors is useless if you cannot get enough data to them on time.

Traditional very large-scale integration (VLSI) design uses an ad hoc and monolithic communication fabric that connects different resources [such as, for example, the memory and the arithmetic logic unit (ALU)] on the chip together by dedicated wires. With increasing system complexity and the continuing miniaturization of the technology, radically new interconnect approaches will be necessary if we want to sustain the current pace of progress. ${ }^{9}$ Two main factors potentially limit performance: ${ }^{10,11}$ (1) The miniaturization of wires, unlike transistors, does not enhance their performance, which is why wires are now more important than transistors. ${ }^{9}$ (2) Global wires that communicate signals across the whole chip increase delays and therefore limit the system scalability. The 2005 ITRS roadmap ${ }^{25}$ (and the 2006 update) lists a more detailed number of critical challenges for interconnects. In recent years, true 3D architectures and associated design methodologies have emerged, which offer an attractive option to address some of the current interconnect challenges. $^{26,27}$

On the other hand, $\mathrm{NoC}^{28,29}$ have been proposed as a promising solution to address the on-chip communication challenges and to cope with the increasing communication requirements. The basic idea behind this paradigm is that the different modules on the chip (e.g., IP cores) are interlinked by a bus-like communication network with programmable switch blocks that support packet-oriented traffic. Thus, NoC architectures decouple the communication fabric from the processing and storage elements ${ }^{29}$ and provide a more modular view of the system, which allows to better master complexity of large-scale systems, to decompose it into independent subsystems, and to keep things flexible. The additional communication fabric obviously results in an overhead of area and energy dissipation, which the designer has to consider in addition to all other design tradeoffs. The overhead largely depends on the connectivity, the number of switch blocks, their complexity, and the number of possible repeaters. Pande et al. provide some estimates for their framework. ${ }^{30}$

The NoC approach is very general and allows for any interconnect architecture between functional and communication blocks, such as, for example, local and regular, fattree, hypercube, irregular and application specific, or smallworld topologies as described in Sec. II A (see Refs. 29 and 30 for some examples). Field-programmable gate arrays (FPGAs), for example, offer a regular arrangement of programmable logic blocks that are interconnected by a programmable communication fabric, which introduces a great degree of freedom for the application designer. However, once the NoC topology is selected, the only remaining degree of freedom is the routing strategy.

In the following, we will make use of the NoC paradigm in combination with nature-inspired 3D network topologies for our explorative framework. Very recently, other researchers investigated the idea of inserting long-range connections to otherwise regular NoC. Ogras et al. ${ }^{31}$ showed that a significant reduction in the average packet latency can be achieved by superposing a few long-range links to a standard mesh network. In their approach the links are, however, not inserted at random but where they are most useful. Oshida and Ihara $^{32}$ investigate the performance of scale-free network-on-chip topologies. Their findings show that short latencies and a low packet loss ratio can be achieved. The parallel processing community has also looked into improving large-scale multicomputer interconnects by adding shortcuts to bypass nodes. ${ }^{33,34}$ Fukś and Lawniczak ${ }^{35}$ and Lawniczak et al. ${ }^{36}$ examined more generally how the introduction of 


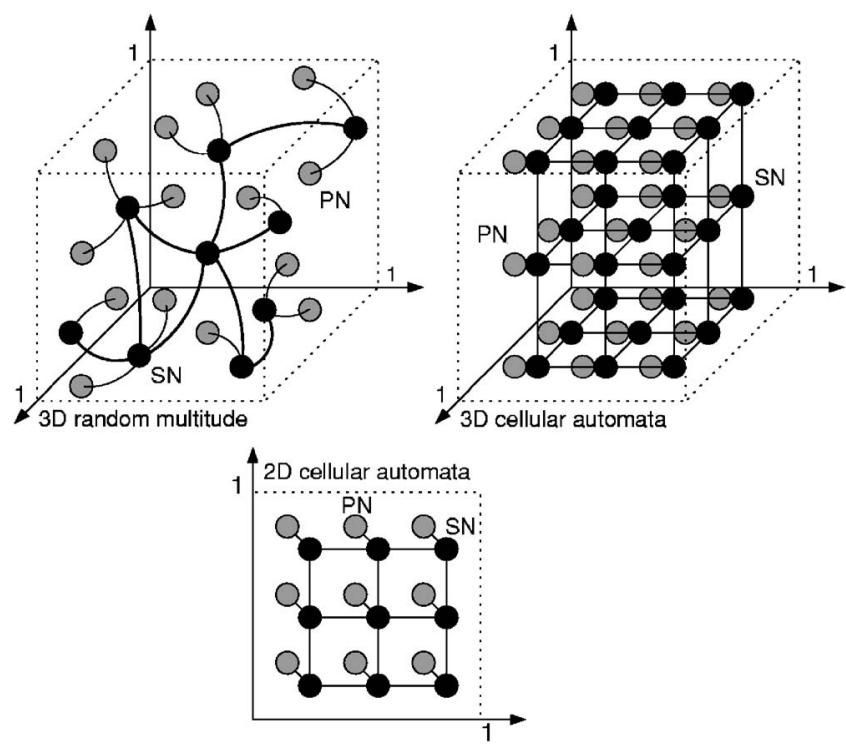

FIG. 1. Top left: A 3D random multitude (RM) example composed of processing nodes (PNs), switch nodes (SNs), and interconnections. Top right: A 3D CA grid-like architecture. Bottom: A 2D CA grid-like architecture.

additional random links influences the performance of computer networks. For all these approaches, the performance strongly depends on the routing algorithm and whether it is able to efficiently use the provided "shortcuts" in the networks while avoiding congestion at the same time.

\section{DESCRIPTION OF THE FRAMEWORK}

We are interested in experimentally exploring selfassembled networks-on-chip architectures that are built in a largely random manner. If we want nanoscale electronics to become a success, we have to show that we can (1) build systems that involve an Avogadro number of components and (2) that such a system can efficiently and robustly solve a specific task. In the absence of mathematical models for self-assembled electronics (such as, for example, nanowire growth models), we decided to build a toy framework that would allow us to experimentally explore the properties and design tradeoffs we are interested in. The framework also allows us to quantitatively compare the irregular and selfassembled with representative and regular nearest-neighbor fabrics.

In the following sections, the network-on-chip-like framework and the evaluation methodology, which is inspired by Pande et al. ${ }^{30}$ shall be described in more details.

\section{A. Node and link model}

The basic system-on-chip-like architecture that we use is composed of (1) programmable computing elements, called processing nodes (PNs); (2) an associated switch-based interconnect fabric, which is itself composed of (3) switch nodes (SNs); and (4) bidirectional point-to-point interconnects among them. Both processing and switch nodes can be considered as simple modules of a large-scale system that needs to communicate efficiently among each other. Figure 1 shows there different arrangements. The interconnect topologies shall be described in the next section.

Each switch node can only transmit in parallel messages on $C$ different virtual channels to its neighbors (see, e.g., Ref. 30 for more details about the concept of virtual channels) according to a specific routing scheme. No further information processing is done in the switch nodes. We assume that they can temporarily store a limited number of $M$ messages. For the sake of simplicity, we have chosen this number to be large enough, i.e., $M=100$, to handle our simulations without creating jamming and losing messages. The processing nodes, on the other hand, simply send and receive messages according to a specific traffic scheme. Since we are interested in interconnect issues here, we do not further specify or limit the processing nodes' computing capacity.

\section{B. Network topologies}

We have decided to compare the following six reference network topologies in order to quantitatively evaluate the self-assembled and irregular fabrics:

- 2DCA. Two-dimensional (unfolded) regularly arranged and locally interconnected (von Neumann neighborhood) (see Fig. 1);

- 3DCA: Three-dimensional (unfolded) regularly arranged and locally interconnected (six neighbors per switch node) (see Fig. 1);

- 3DRMStandard: Three-dimensional random arrangement, small world, power law, $\alpha=1.8$;

- 3DRMLocal: Three-dimensional random arrangement, small world, power law, $\alpha=3$ (locally interconnected);

- 3DRMGlobal: Three-dimensional random arrangement, small world, power law, $\alpha=0$ (globally interconnected);

- 3DRMRealistic: Three-dimensional random arrangement, small world, power law, $\alpha=1.8$, upper limit $k_{\max }$ on the number of connections per node, independently of the average connectivity.

We call a 3D random arrangement a random multitude (RM). Figure 1 depicts a 3D random multitude, a 2D CA, and a 3D CA topology. We do not use folded versions for the cellular-automata-like topologies because that would require long-distance connections. For both 2D and 3D CAs, the processing nodes are regularly arranged in the $2 \mathrm{D}$ or $3 \mathrm{D}$ Euclidean space inside a unitary square, respectively, cube. The number of processing nodes is equal to the number of switch modes, and each processing node is connected to its associated switch node by a single connection of 0.01 unit length.

For the random multitude, both processing and switch nodes are randomly arranged in 3D space, as illustrated in Fig. 1 (top left). Both the arrangement and the wire topology are inspired by self-assembled nanoscale electronics, as we will see in Sec. III E. To make comparisons with the CA grid-like architectures easier, we assume that each processing node is connected to the nearest switch node by a single connection only. The switch nodes are connected among themselves by a small-world power-law network ${ }^{15,16,19}$ with average connectivity $\left\langle k_{S}\right\rangle$, i.e., each switch node establishes 


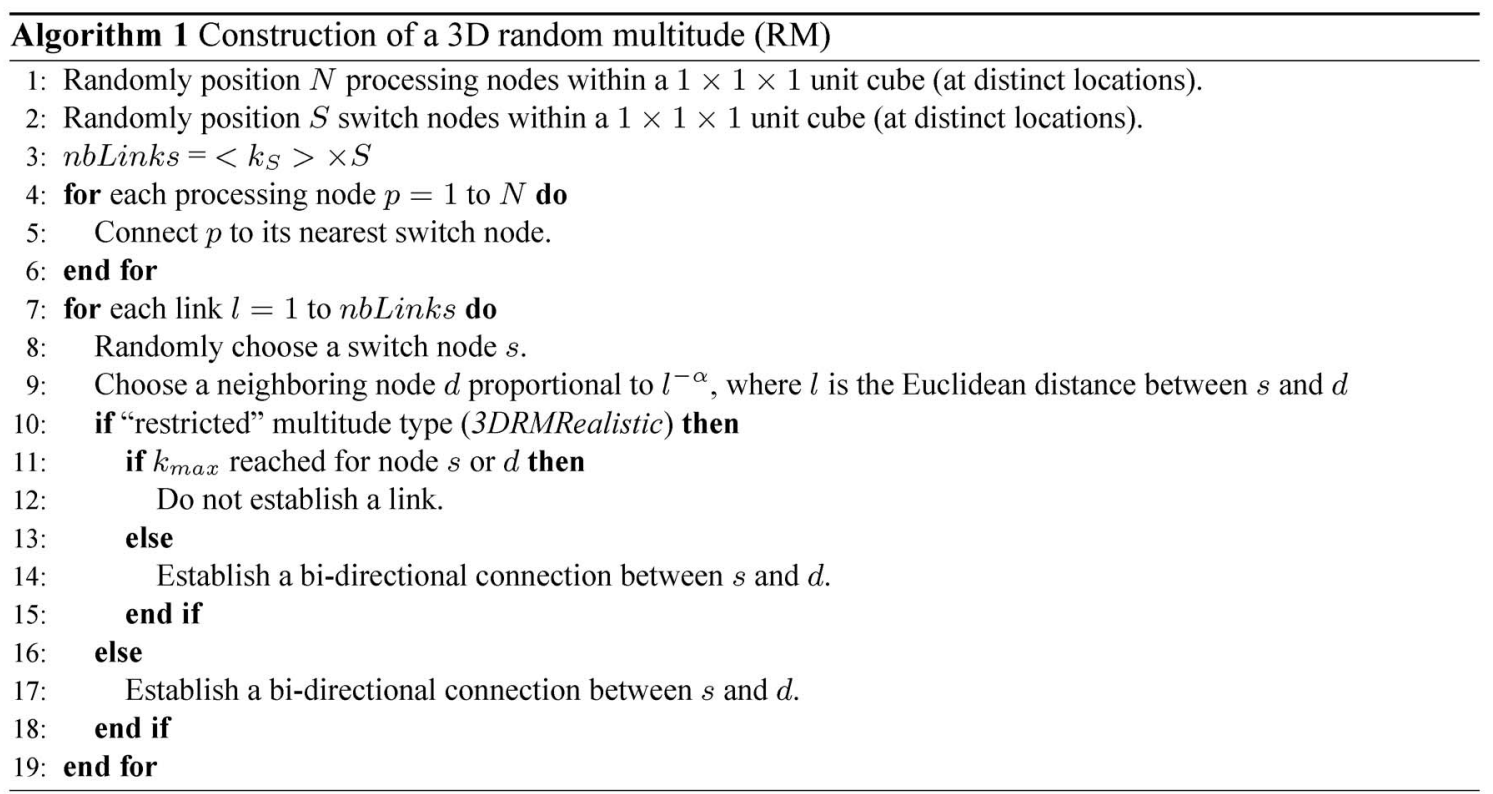

$\left\langle k_{S}\right\rangle$ connections on average with its neighbors proportional to $l^{-\alpha}$, where $l$ is the Euclidean distance between the two switch nodes in question. Thus, the bigger $\alpha$, the more local the connectivity. For $\alpha=0$, we obtain the original WattsStrogatz small-world topology. The choice of $\alpha=1.8$ was guided by the experiments and will be further explained in Sec. IV B. For all our experiments, we also make sure that the graphs do not contain disconnected parts.

Algorithm 1 summarizes the construction of a random multitude with average connectivity $\left\langle k_{S}\right\rangle$ from an algorithmic point of view. For the restricted version (3DRMRealistic), there is an upper limit $k_{\max }$ on the maximum number of connections per node that can be established, the idea being that this realistic restriction is to make the topology more physically plausible. More details shall be given in Sec. III E.

\section{Routing and traffic models}

Once a topology is chosen and fixed, the only free parameter available is essentially the routing strategy, which plays a crucial role for the overall system performance. There exists a large number of different routing strategies and flavors, which allow to send packets along prespecified or dynamically chosen paths in a given network from a source to a destination. Whether throughput or latency or any other property needs to be maximized, highly depends on the application. In general, an ideal routing algorithm is adaptive, decentralized, robust, and guarantees quality of service (QoS) within well-defined bounds.

Here, we deal with packet routing only and the switch nodes do therefore have to know where to route a packet that they receive. The path information can be obtained dynamically or statically, based on the available information on the network's topology. Shortest path routing is frequently used, but it is not necessary the best routing strategy ${ }^{37}$ since congestion has to be taken into account.

Efficient search and information transfer on complex networks while avoiding congestion and optimizing through- put and latency at the same time are of great importance in real-world systems. ${ }^{38-44}$ It has also been shown that smallworld and scale-free networks offer great communication characteristics, are efficient to navigate, ${ }^{18,45}$ and reduce congestion. $^{46,47}$ Routing based on local information only (e.g., Refs. 40 and 45) is of particular interest for large-scale systems where global path information is either not available or too costly to store in each node.

Here, we are more interested in exploring the extrema than to use any complicated and highly optimized routing algorithm. We use two main routing strategies: (1) shortest path routing and (2) random wandering. Shortest path routing is optimal if the traffic is low and no congestion occurs, but every node needs to store a routing table that can get considerably large for large networks. In random wandering, the switch node that receives a message simply sends it to a randomly chosen neighbor. This is very simple to implement and robust against link and node failures, but very inefficient for larger system sizes. We have also explored ant routing ${ }^{48}$ as an alternative, which essentially allows to build shortest paths in a decentralized manner by the messages (i.e., the "ants") themselves.

We decided to adopt a very simple - and admittedly not very realistic - traffic model that is, however, widely used to evaluate networks: uniform random traffic. Every processing node $n$ injects a message to a randomly chosen processing node into the network with probability $p_{I}$ at each time step. If this injection rate $p_{I}$ is 1 , a message will be generated during every time step by every node.

\section{Performance metrics}

To compare the different network-on-chip topologies, we use standard performance metrics and an evaluation methodology inspired by Pande et al. ${ }^{30}$ While area overhead and especially energy consumption are increasingly important, we are not focusing on these aspects here. We are mainly concerned by (1) the average number of hops a message has to take in the shortest path from a source to a destination, 
i.e., the number of switch nodes in the path; (2) the latency; and (3) the shortest path length (in distance units). Although throughput is important, we assume that for our applications, we have low traffic that does not lead to congestion.

The average number of hops is measured over $T$ simulation time steps and over all messages sent. The average shortest path length is measured in distance units and takes into account the paths between all possible combinations of processing nodes. The throughput of a switch node is measured in messages per number of updates per switch node.

Unless otherwise stated, our experiments used $S=N$ $=64$, a synchronous node update, six virtual channels per node (i.e., a 3D CA-node could send a message into all directions simultaneously), an average switch node connectivity of $\left\langle k_{S}\right\rangle=6$, an exact processing node connectivity of 1 , a traffic injection rate of $p_{I}=0.1$ (i.e., each node sends a message every $10^{\text {th }}$ time step on average), and a maximum connectivity of $k_{\max }=10$ for the realistic multitude 3DRMRealistic. In our framework, a message is an abstract entity and we do not take into account its size in terms of number of bits.

\section{E. Physical plausibility and realizability}

There exists an abundance of abstract computing models that are either hard or impossible (e.g., when infinite resources or time are involved) to physically realize. Building computers is about hijacking the underlying material in order to make it do the things we want. Today, the vast majority of fabrication processes is top-down oriented and given a computing architecture, the goal is to successfully realize it, for example, by using silicon-based electronics. However, with the ongoing miniaturization and the constant need for more computing power, there has been an increasing interest in bottom-up assembling techniques and disruptive new computing concepts and methodologies. Especially, selfassembling molecular electronics, based, for example, on nanowires or nanotubes, bear unique challenges and opportunities for new paradigms.

Despite important progress, the fabrication of ordered 3D hierarchical nanostructures remains very challenging. ${ }^{49}$ Here, we argue that because of fewer physical constraints, computing architectures that are "assembled" in a largely random manner are easier and cheaper to build than highly regular architectures, such as crossbars or cellular-automatalike assemblies, which usually require a perfect or almost perfect establishment of the connections. Self-assembly, for example, is particularly well suited for building random structures. ${ }^{13}$ Power-law connection-length distributions have been observed in many systems created through selforganization, such as the human cortex or the Internet, and they can be considered "physically realizable." ${ }^{16}$ Such topologies evolve naturally in nature, essentially because of the cost associated with long-distance connections, which prevents a uniform wiring probability over all nodes.

There is very little work about computing architectures with irregular assemblies of connections and components. Tour et al., ${ }^{6}$ for example, explored the possibility of computing with randomly assembled, easily realizable molecular switches, that are only locally interconnected, however. On the other hand, Hogg et al. ${ }^{50}$ present an approach to build reliable circuits by self-assembly with some random variation in the connection location. With the exception of a few researchers (e.g., Refs. 4, 6, and 51), the vast majority working in the field of nanoscale electronics tries to build regular structures, which allow for a more or less straightforward mapping of higher-level computing architectures. Computing with highly irregularly assembled physical substrates is undoubtedly a new and disruptive paradigm. The main question we would like to address to some extent in this section is whether and how the framework as described above could be physically implemented.

Designing nanoscale interconnects is guided by a number of major and dependent tradeoffs: (1) the number of long(er)-distance connections, (2) the physical plausibility, and (3) the efficiency of communication. Since the fabrication of nanoscale computing architectures tends to be very challenging, we opt for a fabrication-friendly approach and will try to live with what we can currently build. Although we are unable to provide experimental results at this point, plausible approaches for physical realizations shall be briefly sketched here. Preliminary experiments with both nanowires and nanotubes are currently underway at the Los Alamos National Laboratory.

We believe that a random multitude would be best realized in a hybrid way today, where the processing and switch nodes are, for example, made of current (nanoscale) silicon. Since we are focusing on the interconnections here, we do not further specify the characteristics of these nodes. Our only intention is to keep both the computation and the routing as simple as possible to minimize the node's complexity. The interconnect fabric would then be gradually selfassembled using either nanowires or nanotubes. ${ }^{52} \mathrm{We}$ imagine that in a first step, both the processing and the switch nodes would either be randomly placed in a scaffolding structure or be submerged in some kind of fluid, similar to the self-assembly of nanowires from a solution. ${ }^{53}$ Each processing and switch node will have a limited number of seed points, where either nanowires or nanotubes could connect to or grow out by, for example, a self-catalytic growth process. The wires would grow in random directions and eventually make contact with another switch or processing node. The growth could be guided by additional scaffolding structures or, for example, by magnetic and electrical fields. Ye et al. ${ }^{54}$ for example, present an approach for the directed assembly by means of electrodeposition or vapor deposition. As an alternative to directly growing wires from seed points on the nodes, one could also imagine to prefabricate the wires and then to connect them in a second step to the nodes, for example, while being immersed in a solution.

To obtain a specific power-law distribution of connection lengths, and thus to obtain a small-world topology as described above, one could imagine to grow different wires with different probabilities, whose lengths follow a powerlaw distribution. Alternatively, if the wires directly grow out of the nodes and randomly connect to neighboring nodes, we hypothesize that it is possible to obtain a desired distribution as a function of the Euclidean distance between the nodes by 
imposing restrictions on the wire growth lengths. However, physical wire growth models or experimental results would be necessary to further investigate this option. Note also that current nanowires tend to be rather short because of a high resistance and the probability of breaks, which will naturally limit the number of long-distance connections.

In order to make our framework as realistic as possible, we introduced in Sec. III B a limitation, $k_{\max }$, on the number of links that a node can carry for the 3DRMRealistic random multitude. Given the above growth mechanisms, this is a realistic assumption because one cannot assume an unlimited number of contacts on a given node. The exact value of $k_{\max }$ will depend on the wire type and the fabrication technology. For all our experiments, we use a value of $k_{\max }=10$, which seems rather pessimistic but allowed to make a plausible comparison with the unrestricted random multitude, 3DRMStandard.

In summary, we believe that there exist several promising paths to physically realize irregularly self-assembled networks of wires and nodes with a specific topology. The random multitude construction algorithm (see Sec. III B) of our framework is meant to reflect what we could possibly assemble in reality in the very near future.

\section{EXPERIMENTS}

In the following sections, we will perform a number of pragmatic and simple experiments with the goal to compare the performance of realistic, both regular and irregular, network-on-chip topologies as presented in Sec. III B. All simulations were written in Matlab.

\section{A. Experiment 1: System scalability}

The goal of this first experiment is to illustrate how the different topologies perform as the system size scales up. What works for $N=64$ nodes does not necessary work for $N=10000$ nodes. As we have seen before, scalability is a critical issue for nanoscale systems because it is generally very easy to build systems that involve huge numbers of components, e.g., Avogadro-scale systems. If the communication fabric does not allow to efficiently send data across such an assembly, it will be impossible to solve tasks efficiently and thus to stay competitive with conventional design approaches.

For all six assemblies as described in Sec. III B, we have varied the system size and measured the average number of hops, which is proportional to the average path length $L$, i.e., the number of edges in the shortest path between two nodes, averaged over all pairs of nodes as, for example, used in Ref. 14. The different system sizes we used were: (1) $N=S$ $=[9,14,19,24,29,34,39,44,49,54,59,64]$ for random multitudes; (2) $N=S=[9,16,25,36,49,64,81,100,121]$ for 2D CAs; and (3) $N=S=[8,27,64,125]$ for 3D CAs. Shortest path routing was used.

As Fig. 2 shows, the locally connected topologies, i.e., the 2D and 3D CAs as well as the local 3D random multitude, scale up worse with system size than the other three topologies. Not surprisingly, the globally connected random multitude $(\alpha=0)$ scales up best because of the uniform re-

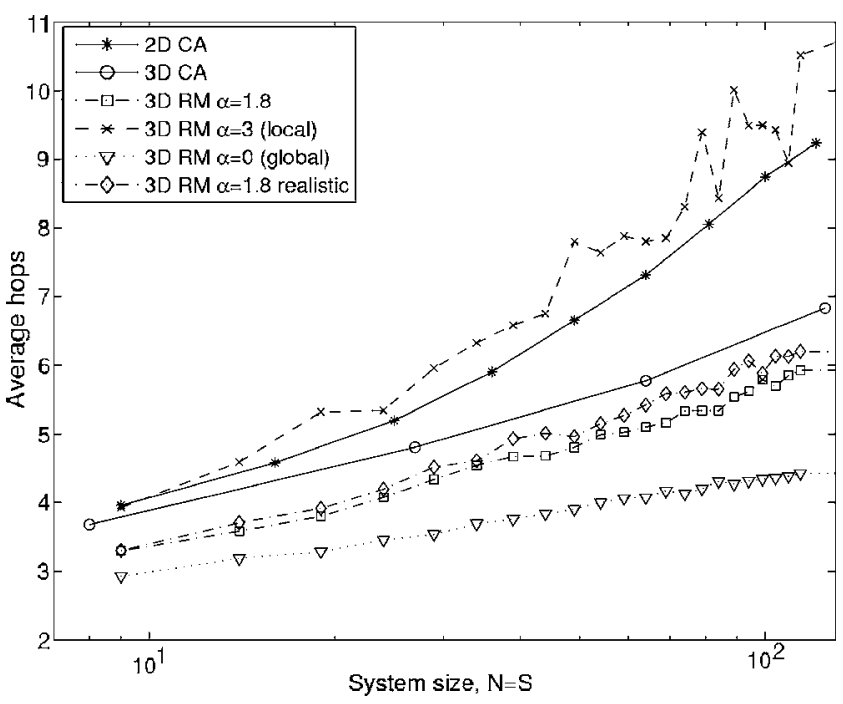

FIG. 2. Scaling of the number of hops as a function of the system size $N$ $=S$. The $\alpha=0$ (global), $\alpha=1.8$ (standard), and $\alpha=1.8$ (realistic) random multitudes show a logarithmic scaling behavior. Average over 10 runs for RMs and over 7 runs for CAs.

wiring probability over all nodes, independently of the Euclidean distance between them. The average path lengths of small-world graphs scale up logarithmically with the number of nodes, which Fig. 2 confirms. Note that there is only a very small difference between the realistic random multitude and the unrestricted $\alpha=1.8$ multitude. This is good news and illustrates that the limited, and thus more realistic, connectivity has little effect on the average number of hops as the system is scaled up.

\section{B. Experiment 2: Local versus global connections}

The distribution of the long- and short-distance connections as a function of the Euclidean distance between the nodes is a crucial parameter in our model. Clearly, we are interested in a great network performance while having a minimal number of global connections, which are generally costly to establish. In this experiment, we explore the network's communication characteristic as a function of the parameter $\alpha$ and compare it with the fixed 2D and 3D CA grid-like assemblies. The function $l^{-\alpha}$, where $l$ is the Euclidean distance between the two switch nodes in question, describes the connectivity of the random multitudes.

Figure 3 shows the average number of hops as a function of the power-law exponent $\alpha$. The 2D and 3D CAs, as well as the locally connected and fixed random multitude $(\alpha=3)$, are plotted as a reference, although their value is independent of $\alpha$. As one can see, the number of hops increases dramatically the bigger $\alpha$ gets, i.e., the more local the connectivity becomes. For comparison, the one-dimensional (1D) ring structure as used in the original Watts-Strogatz rewiring model $^{14}$ is also shown. The 1D ring structure performs worse with increasing $\alpha$ than the 3D random multitudes, which offer a higher connectivity. For a value of $\alpha$ that is slightly smaller than 2, both the unrestricted and the realistic random multitudes perform better than the 3D regular assembly. 


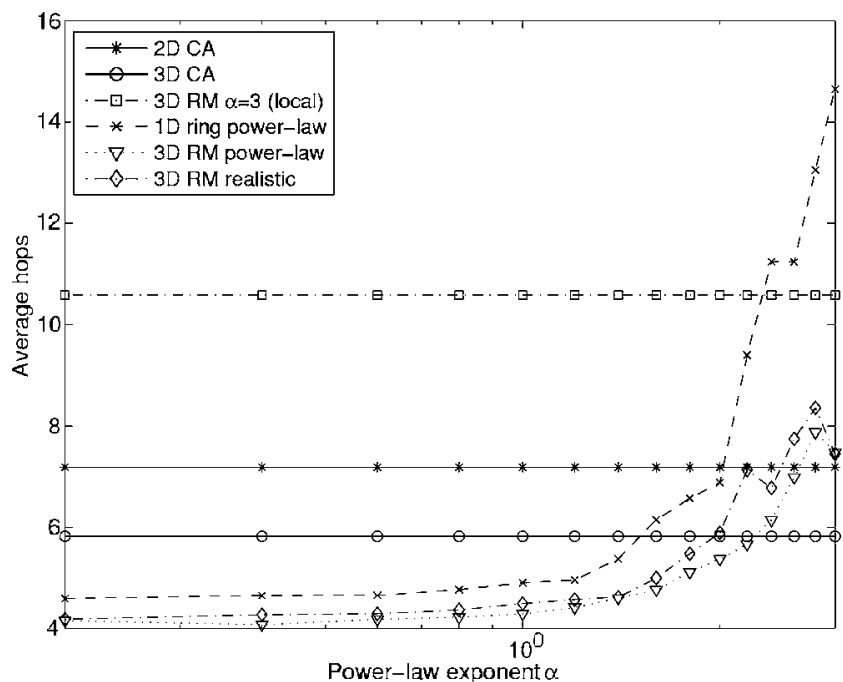

FIG. 3. The average number of hops as a function of the power-law exponent $\alpha$. Average over 2 runs, shortest path routing, $N=S=64$.

In our framework, the average latency is essentially proportional to the average number of hops because we keep the traffic injection rate low to avoid jamming. Figure 3 also confirms the small-world characteristic of the wiring, where the average path length - the average number of hops in our case-drastically drops when a few global connections are added (i.e., when $\alpha$ becomes smaller). Figure 4 shows the clustering coefficient $C$ (Ref. 14) of the 1D ring and the two $3 \mathrm{D}$ random multitudes. The random multitudes have a very low clustering coefficient, while the $1 \mathrm{D}$ ring behaves like the Watts-Strogatz model.

As Petermann and De Los $\operatorname{Rios}^{16}$ find both analytically and numerically, the small-world phenomena in a network built using a power-law decaying distribution of shortcut lengths occurs when $\alpha<D+1$, where $D$ is the network's dimension. In the case of our random multitudes, $D=3$, which confirms our observations of small-world behavior. Based on the results as shown in Fig. 3, we have chosen

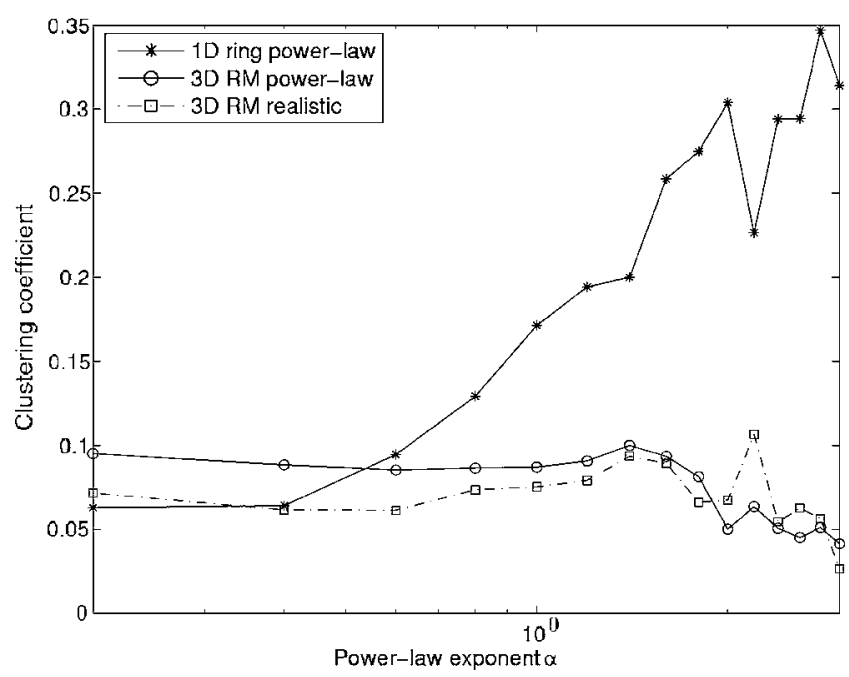

FIG. 4. The clustering coefficient as function of the power-law exponent $\alpha$. Average over 2 runs, shortest path routing, $N=S=64$.

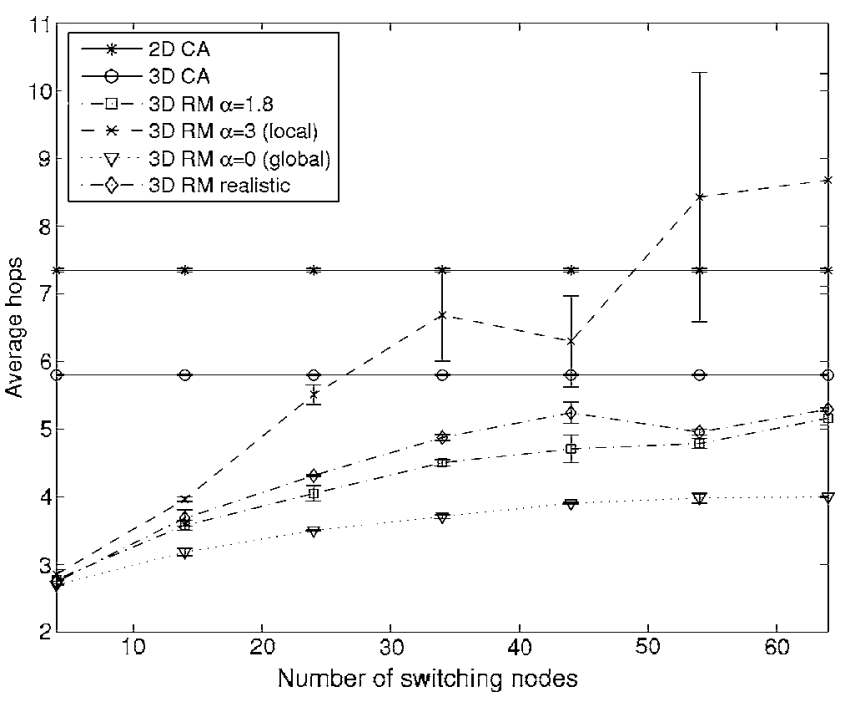

FIG. 5. The average number of hops as a function of the number of switch nodes $S$. Average over 2 runs, shortest path routing, $N=64$.

$\alpha=1.8$ for the regular random multitudes (3DRMStandard and 3DRMRealistic) for the following experiments.

\section{Experiment 3: Number of switch nodes and connectivity}

From a design perspective, one is obviously interested in minimizing the number of switch nodes and the connectivity among the switch nodes to a level that does not significantly affect the system performance. In this experiment, we explore the framework's characteristics by varying the number of switch nodes $S$ and the switch node connectivity $\left\langle k_{S}\right\rangle$, while keeping $\alpha$ constant.

Figure 5 shows that the average number of hops for a message to take on the shortest path from any source to any destination increases with the number of switch nodes $S$. The fixed CA topologies are shown for comparison. However, there is a tradeoff between the number of hops and the throughput a network can handle. A low number of switch nodes limits the throughput, which we cannot illustrate here because we have chosen a low traffic injection rate that avoids jamming. Thus, depending on what the network needs to be optimized for (i.e., lower number of hops, throughput, short average path length, etc.), one can make the appropriate choice for the number of switch nodes. Obviously, the amount of hardware resources and the volume required will also come into play in reality.

Figure 6 shows that the higher the switch node connectivity $\left\langle k_{S}\right\rangle$, the lower the average shortest path length. The fixed CA topologies are shown for comparison. Once again, one can observe only a small difference between the unrestricted and the realistic random multitude. Further results shall be summarized as follows:

- A higher switch node connectivity decreases both the average latency and the average number of hops. The throughput is only slightly improved;

- The higher the number of switch nodes $S$, the higher the number of hops and the higher the average latency. The 


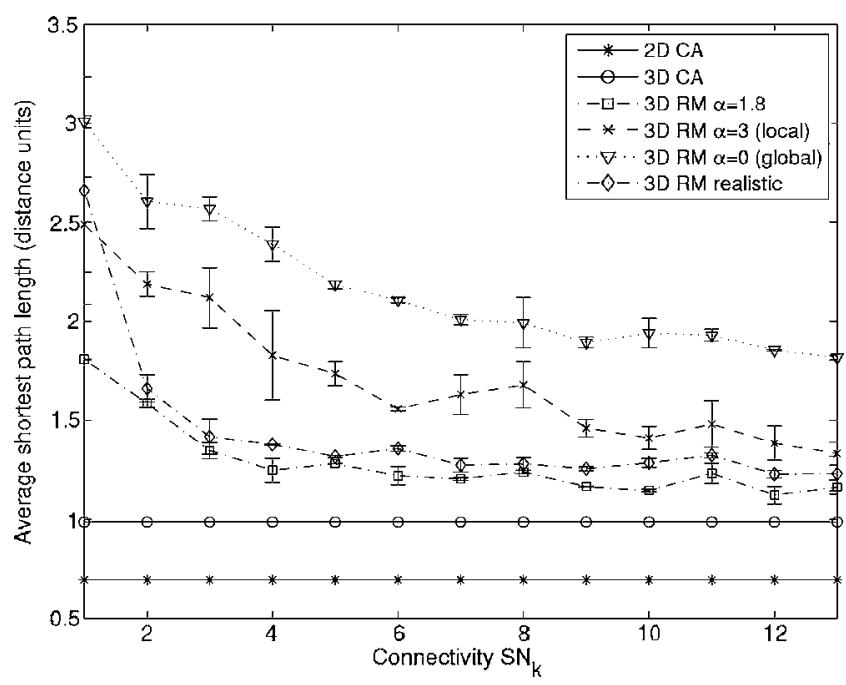

FIG. 6. The average path length as a function of the average switch node connectivity $\left\langle k_{S}\right\rangle$. Average over 2 runs, shortest path routing, $N=S=64$.

lower $S$, the higher the average path length and the higher the throughput;

- The higher the number of virtual channels $C$, the higher the node throughput (within the limits of the capacity of the physical links) and the lower the average latency. The average shortest path length is not affected by $C$.

We can conclude that there are no "optimal" values for connectivity, the number of switch nodes, and the number of virtual channels. Instead, choosing the right values is a matter of dependent tradeoffs in the design space. Local connections are very interesting from an implementational point of view, but offer reduced global communication characteristics only, which directly affects the efficiency of problem solving (see also Sec. IV E). Adding a few long(er)-distance connections proportional to the distance between the nodes is physically plausible and greatly improves the overall system performance as well as the robustness, as we will see in the next section.

\section{Experiment 4: Robustness against link failures}

Robustness against manufacturing defects and dynamic failures is critical for future Avogadro-scale self-assembled nanoscale architectures. ${ }^{55}$ Due to the small feature sizes, such systems are generally expected to be much more prone to radiation-induced soft errors, to thermal noise, ${ }^{2}$ and to fabrication defects because of the self-assembly processes.

In order to compare the robustness against link failures of both regularly and irregularly interconnected topologies, we randomly removed links between the switch nodes in our six reference assemblies. It is well known that small-world and scale-free networks are robust against random failures of nodes and links. ${ }^{15,56}$ As Fig. 7 illustrates, this is also valid for our framework. The average number of hops is plotted as a function of the number of removed switch links. In this experiment, we used random wandering to illustrate an extreme case. As one can see, both 2D and 3D CA topologies start to perform worse, i.e., the average number of hops increases,

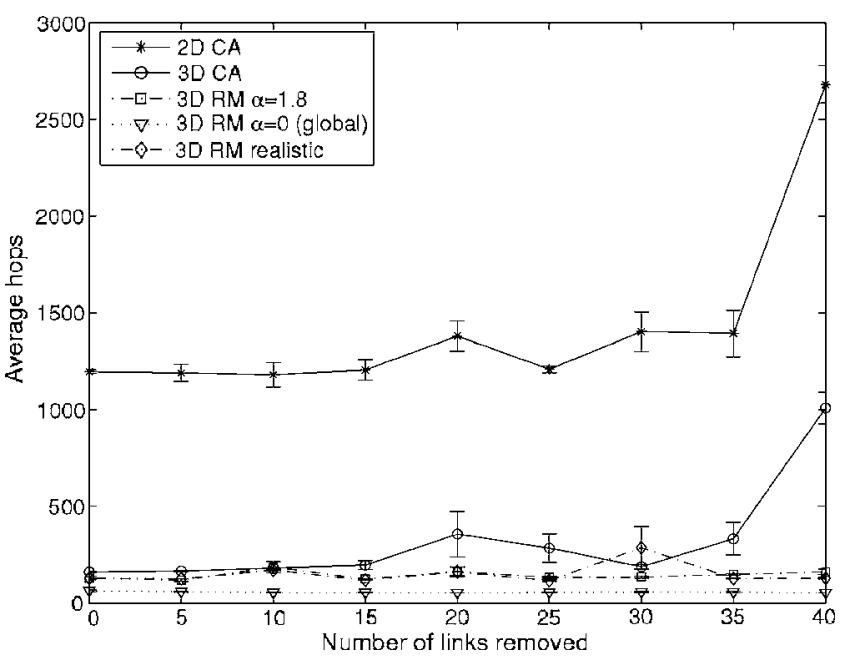

FIG. 7. The average number of hops as a function of the number of randomly deleted links. Average over 2 runs, random wandering, $N=S=64$.

when the number of randomly removed links approaches 40 , while the random multitudes essentially remain unaffected by the removed links.

The random link failures admittedly represent a well oversimplified fault model; nevertheless, it illustrates that the irregular small-world topologies of our framework provide "robustness for free" to some extent, even without using any specific fault detection and isolation technique.

\section{E. Experiment 5: Solving a simple task}

In this last experiment, we are interested in evaluating the performance of solving a simple problem, which is well known in the area of cellular automata: the synchronization task. This "global" task is essentially trivial to solve if one has a global view on the entire system (i.e., if one has access to the state of all nodes at the same instant in time), but it is nontrivial to solve for locally connected cellular automata or random boolean networks (RBNs). Although it is commonly called a "toy problem," the synchronization task has actually various real-world applications, such as, for example, in sensor networks, where one cannot assume global synchronization and global signals, and thus special mechanisms are required. ${ }^{57}$

The synchronization task (also called firefly task) for synchronous CAs was introduced by Das et al. ${ }^{58}$ and studied among others by Hordijk ${ }^{59}$ and Sipper. ${ }^{60}$ In this task, the two-state $D$-dimensional automaton, given any initial configuration, must reach a final configuration within $M$ time steps, that oscillates between all $0 \mathrm{~s}$ and all $1 \mathrm{~s}$ on successive time steps. The whole automaton is then globally synchronized.

Here, we use a slightly adapted version the task for our framework: we assume that each processing node in our framework contains an oscillator, which frequency is specified by a number between $0 \leqslant f_{\text {osc }} \leqslant 1$. The modified task then consists of finding a common frequency for all oscillators. The algorithm is inspired by the averaging algorithm as described in Ref. 57. Each processing node state is initialized to a random value from the interval $[0,1]$ before it repeatedly 


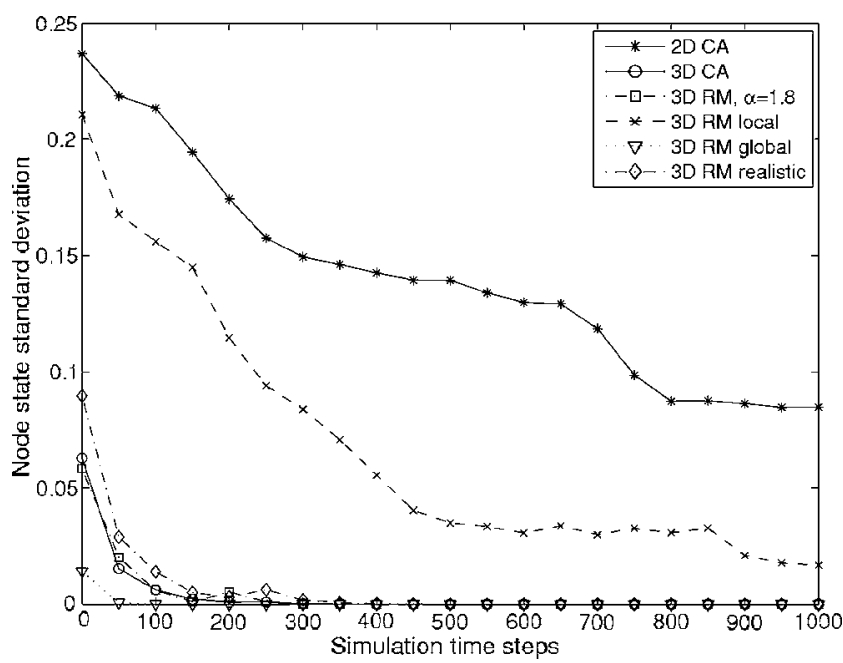

FIG. 8. Performance of the synchronization task. The smaller the standard deviation of the node state values, the better the nodes are synchronized. The initial values for each curve depend on the randomly initialized network state. $N=S=64$, random wandering.

performs the following steps in an asynchronous manner: (1) send current oscillator frequency to a random processing node; (2) if the current node $i$ receives a message from any other processing node $r$, then average own oscillator $f_{i}$ with neighbor frequency $f_{r}$; (3) set own oscillator to this frequency $f_{i}=f_{i}+f_{r} / 2$; and (4) also send it to a new random processing node.

There are obviously numerous (also more efficient) ways to solve this task, but here we are interested in an illustrative comparison rather than in the absolute performance values and limits. We compared how this simple algorithm performed on the investigated interconnect fabrics by using random wandering. As Fig. 8 illustrates, the small-world topologies perform best. Both the $2 \mathrm{D}$ and the locally interconnected multitude very slowly converge because of the poor global communication characteristics. Not surprisingly, the globally connected random multitude performs best.

It has been shown elsewhere that irregular small-world interconnects perform better on both the synchronization (e.g., Refs. 24 and 61-63 and many others) and the density classification task (e.g., Ref. 24) than purely locally interconnected topologies. However, the frameworks and assumptions used in each approach are somehow different and sometimes not straightforward to compare. The results of our framework merely confirm what has been found theoretically elsewhere and in our two previous experiments, namely that the excellent communication characteristics (i.e., short characteristic path length, the small latency, etc.) also helps to efficiently solve tasks, especially tasks which require a lot of global communication. From an evolutionary perspective, this also seems the reason why most natural networks, e.g. the brain, ${ }^{20,21,64}$ have evolved with the small-world and scale-free property. The relationship between efficient problem solving and interconnection topologies has naturally also preoccupied the parallel computing community since its beginning (e.g., Ref. 65).

\section{CONCLUSION}

We have experimentally investigated in a pragmatic way several relevant metrics of both regular and irregular, realistic system-on-chip-like computing architectures for selfassembled nanoscale electronics; namely, 2D and 3D localneighborhood as well as irregularly built small-world interconnects with different distributions of long-distance connections. The small-world architectures with a power-law decaying distribution of shortcut lengths investigated in our framework are both physically plausible, could likely be built very economically by self-assembling processes, possess great communication characteristics, and are robust against link failures. While regular and local-neighborhood interconnects are easier and more economical to build than interconnects with lots of global or semiglobal long-distance connections, we have seen in the previous section that they are not as efficient for global communication, which is very important and directly affects how efficient problems can be solved with such architectures. Small-world networks with a uniform distribution of long-distance connections or pure Erdös-Rényi random networks, on the other hand, are not physically plausible because one has to assume an increasing wiring cost with distance. As our results have shown by means of a simplistic, yet realistic framework, small-world topologies with a power-law decaying distribution of shortcut lengths offer a unique balance between performance, robustness, physical plausibility, and fabrication friendliness. In addition, it has been shown that adaptive routing-which we have not explored in detail here-is very efficient on small-world power-law graphs. ${ }^{18,45}$

We believe that computation in random self-assemblies of components and interconnections (see, e.g., Refs. 4, 6, 50, and 51) is a highly appealing paradigm, both from the perspective of fabrication as well as performance and robustness. This is obviously a radically new technological and conceptual approach with many open questions. For example, there are essentially no methodologies and tools that would allow (1) to map an arbitrary computing architecture or a logical system on a randomly assembled physical substrate; (2) to do arbitrary computations with such an assembly; and (3) to systematically analyze performance and robustness within a rigorous mathematical framework. There are also many open questions regarding the self-assembling fabrication techniques, which will need to be further explored in the future.

\section{ACKNOWLEDGMENTS}

The author is very grateful for the fruitful discussions with Elshan A. Akhadov, Steven K. Doorn, Sandip Niyogi, Tom Picraux, Evsen Yanmaz, and Hsing-Lin Wang.

${ }^{1}$ G. E. Moore, "Cramming more components onto integrated circuits," Electronics 38, 114-117 (1965).

${ }^{2}$ L. B. Kish, "End of Moore's law: thermal (noise) death of integration in micro and nano electronics," Phys. Lett. A 305, 144-149 (2002).

${ }^{3}$ J. R. Heath, P. J. Kuekes, G. S. Snider, and R. S. Williams, "A defecttolerant computer architecture: opportunities for nanotechnology," Science 280, 1716-1721 (1998).

${ }^{4}$ J. P. Patwardhan, C. Dwyer, A. R. Lebeck, and D. J. Sorin, "NANA: a 
nano-scale active network architecture," ACM Journal on Emerging Technologies in Computing Systems 2, 1-30 (2006).

${ }^{5}$ A. DeHon, "Array-based architecture for FET-based nanoscale electronics,” IEEE Trans. Nanotechnol. 2, 23-32 (2003).

${ }^{6}$ J. Tour, W. L. Van Zandt, C. P. Husband, S. M. Husband, L. S. Wilson, P. D. Franzon, and D. P. Nackashi, "Nanocell logic gates for molecular computing," IEEE Trans. Nanotechnol. 1, 100-109 (2002).

${ }^{7}$ Y. Chen, G.-Y. Jung, D. A. A. Ohlberg, X. Li, D. R. Steward, J. O. Jepperson, K. A. Nielsen, J. F. Stoddart, and R. S. Williams, "Nanoscale molecular-switch crossbar circuits," Nanotechnology 14, 462-468 (2003).

${ }^{8}$ M. R. Stan, P. D. Franzon, S. C. Goldstein, J. C. Lach, and M. M. Ziegler, "Molecular electronics: from devices and interconnect to circuits and architectures," Proc. IEEE 91, 1940-1957 (2003).

9J. D. Meindl, "Interconnect opportunities for gigascale integration," IEEE Micro. 23, 28-35 (2003).

${ }^{10}$ R. Ho, K. W. Mai, and M. A. Horowitz, "The future of wires," Proc. IEEE 89, 490-504 (2001).

${ }^{11}$ J. A. Davis, R. Venkatesan, A. Kaloyeros, M. Beylansky, S. J. Souri, K. Banerjee, K. C. Saraswat, A. Rahman, R. Reif, and J. D. Meindl, "Interconnect limits on gigascale integration (GSI) in the $21^{\text {st }}$ century," Proc. IEEE 89, 305-324 (2001).

${ }^{12}$ International Technology Roadmap for Semiconductors (ITRS), Semiconductor Industry Association, http://public.itrs.net (2003).

${ }^{13}$ V. V. Zhirnov and D. J. C. Herr, "New frontiers: Self-assembly in nanoelectronics," IEEE Computer, 34 (1), 34-43 (2001).

${ }^{14}$ D. J. Watts and S. H. Strogatz, "Collective dynamics of 'small-world' networks," Nature 393, 440-442 (1998).

${ }^{15}$ T. Petermann and P. De Los Rios, "Spatial small-world networks, A wiring-cost perspective," e-print arXiv:cond-mat/0501420.

${ }^{16}$ T. Petermann and P. De Los Rios, "Physical realizability of small-world networks," Phys. Rev. E 73, 026114 (2006).

${ }^{17}$ B. Kozma, M. B. Hastings, and G. Korniss, "Diffusion processes on power-law small-world networks," Phys. Rev. Lett. 95, 018701 (2005).

${ }^{18}$ J. K. Kleinberg, "Navigation in a small world," Nature 406, 845 (2000).

${ }^{19}$ S. Jespersen and A. Blumen, "Small-world networks: links with longtailed distributions," Phys. Rev. E 62, 6270-6274 (2000).

${ }^{20}$ O. Sporns, D. R. Chialvo, M. Kaiser, and C. C. Hilgtag, "Organization, development, and function of complex brain networks," Trends Cogn. Sci. 8, 418-425 (2004).

${ }^{21}$ V. M. Eguéluz, D. R. Chialvo, G. A. Cecchi, M. Baliki, and A. V. Apkarian, "Scale-free brain functional networks," Phys. Rev. Lett. 94, 018102 (2005).

${ }^{22}$ R. Ferrer i Cancho, C. Janssen, and R. V. Sole, "Topology of technology graphs: small world patterns in electronic circuits," Phys. Rev. E 64, 046119 (2001)

${ }^{23}$ M. Tomassini, M. Giacobini, and C. Darabos, "Evolution and dynamics of small-world cellular automata," Complex Syst. 15, 261-284 (2005).

${ }^{24} \mathrm{~B}$. Mesot and C. Teuscher, "Deducing local rules for solving global tasks with random Boolean networks," Physica D 211, 88-106 (2005).

${ }^{25}$ International Technology Roadmap for Semiconductors (ITRS), Semiconductor Industry Association (http://public.itrs.net/Common/2005ITRS/ Home2005.htm).

${ }^{26}$ Y. Xie, G. H. Loh, B. Black, and K. Bernstein, "Design space exploration for 3D architectures," ACM Journal on Emerging Technologies in Computing Systems 2, 65-103 (2006).

${ }^{27}$ J. H.Ahn, H. S. Kim, K. J. Lee, S. Jeon, S. J. Kang, Y. Sun, R. G. Nuzzo, and J. A. Rogers, "Heterogeneous three-dimensional electronics by use of printed semiconductor nanomaterials problems," Science 314, 1754-1757 (2006).

${ }^{28}$ Networks on Chips: Technology and Tools, edited by G. de Micheli and L. Benini (Morgan Kauffmann, San Francisco, 2006).

${ }^{29}$ L. Benini and G. de Micheli, "Networks on chips: a new SoC paradigm," IEEE Computer 35, 70-78 (2002).

${ }^{30}$ P. P. Pande, C. Grecu, M. Jones, A. Ivanov, and R. Saleh, "Performance evaluation and design trade-offs for network-on-chip interconnect architectures," IEEE Trans. Comput. 54, 1025-1040 (2005).

${ }^{31}$ U. Y. Ogras and R. Marculescu, "“It's a small world after all:" NoC performance optimization via long-range link insertion," IEEE Trans. Very Large Scale Integr. (VLSI) Syst. 14, 693-706 (2006).

${ }^{32} \mathrm{~N}$. Oshida and S. Ihara, "Packet traffic analysis of scale-free networks for large-scale network-on-chip design,” Phys. Rev. E 74, 026115 (2006).

${ }^{33}$ S. Loucif, M. Ould-Khaoua, and L. M. Mackenzie, "On the performance merits of bypass channels in hypermeshes and $k$-ary $n$-cubes," Comput. J. 42, 62-72 (1999).

${ }^{34}$ W. J. Dally, "Express cubes: improving the performance of $k$-ary $n$-cube inerconnection networks," IEEE Trans. Comput. 40, 1016-1023 (1991).

${ }^{35}$ H. Fukś and A. T. Lawniczak, "Performance of data networks with random links," Math. Comput. Simul. 51, 103-119 (1999).

${ }^{36}$ A. T. Lawniczak, A. Gerisch, and K. Maxie, "Effects of randomly added links on a phase transition in data network traffic models," in Proceedings of the Third DCDIS Conference on Engineering Applications and Computational Algorithms (Watam, Waterloo, Canada, 2003), pp. 384-389.

${ }^{37}$ G. Yan, T. Zhou, B. Hu, Z.-Q. Fu, and B.-H. Wang, "Efficient routing on complex networks," Phys. Rev. E 73, 046108 (2006).

${ }^{38}$ S. Carmi, R. Cohen, and D. Dolev, "Searching complex networks efficiently with minimal information,” Europhys. Lett. 74, 1102-1108 (2006).

${ }^{39}$ B. Danila, Y. Yu, J. A. Marsh, and K. E. Bassler, "Optimal transport on complex networks,” Phys. Rev. E 74, 046106 (2006)

${ }^{40}$ B. Danila, Y. Yu, S. Earl, J. A. Mash, Z. Toroczkai, and K. E. Bassler, "Congestion-gradient driven transport on complex networks," Phys. Rev. E 74, 046114 (2006).

${ }^{41}$ A. T. Lawniczak and X. Tang, "Packet switching network performance indicators as function of network topology and routing algorithms," in Proceedings of the 19th Annual IEEE Canadian Conference on Electrical and Computer Engineering (CCECE), Ottawa, Canada, 7-10 May 2006 (IEEE Press, Los Alamitos, CA, 2006), p. 4.

${ }^{42}$ B. K. Singh and N. Gupte, "Crossover behavior in a communication network," Phys. Rev. E 68, 066121 (2003).

${ }^{43}$ L. Zhao, Y.-C. Lai, K. Park, and N. Ye, "Onset of traffic congestion in complex networks," Phys. Rev. E 71, 026125 (2005).

${ }^{44}$ D. K. Arrowsmith, R. J. Mondragon, and M. Woolf, "Data traffic, topology and networks," in Complex Dynamics in Communication Networks, Understanding Complex Systems, edited by L. Kocarev and G. Vattay (Springer, Berlin, 2005), pp. 127-158.

${ }^{45}$ W.-X. Wang, B.-H. Wang, and C.-Y. Yin, Y.-B. Xie, and T. Zhou, "Traffic dynamics based on local routing protocol on scale-free network," Phys. Rev. E 73, 026111 (2006).

${ }^{46} \mathrm{Z}$. Toroczkai and K. E. Bassler, "Jamming is limited in scale-free systems," Nature 428, 716 (2004).

${ }^{47}$ J. J. Wu, Z. Y. Gao, H. J. Sun, and H. J. Huang, “Congestion in different topologies of traffic networks," Europhys. Lett. 74, 560-566 (2006).

${ }^{48}$ G. Di Caro and M. Dorigo, "AntNet: distributed stigmergetic control for communications networks," J. Artif. Intell. Res., 9, 317-365 (1998).

${ }^{49}$ F. Zhang and H. Y. Low, "Ordered three-dimensional hierarchical nanostructures by nanoimprint litography," Nanotechnology 17, 1884-1890 (2006).

${ }^{50}$ T. Hogg, Y. Chen, and P. J. Kuekes, "Assembling nanoscale circuits with randomized connections," IEEE Trans. Nanotechnol. 5, 110-122 (2006).

${ }^{51}$ J. Lawson and D. H. Wolpert, "Adaptive programming of unconventional nano-architectures," J. Comput. Theor. Nanosci. 3, 272-279 (2006).

${ }^{52} \mathrm{~J}$. Hu, T. W. Odom, and C. M. Lieber, "Chemistry and physics in one dimension: synthesis and properties of nanowires and nanotubes," Acc. Chem. Res. 32, 435-445 (1999).

${ }^{53}$ C. Cheng, R. K. Gonela, Q. Gu, and D. T. Haynie, "Self-assembly of metallic nanowires from aqueous solution," Nano Lett. 5, 175-178 (2005).

${ }^{54}$ H. Ye, Z. Gu, T. Yu, and D. H. Gracias, "Integrating nanowires with substrates using directed assembly and nanoscale soldering," IEEE Trans. Nanotechnol. 5, 62-66 (2006).

${ }^{55}$ A. DeHon and H. Naeimi, "Seven strategies for tolerating highly defective fabrication," IEEE Des. Test 22, 306-315 (2005).

${ }^{56}$ R. Albert and A.-L. Barabasi, "Statistical mechanics of complex networks," Rev. Mod. Phys. 74, 47-97 (2002).

${ }^{57}$ Q. Li and D. Rus, "Global clock synchronization in sensor networks," IEEE Trans. Comput. 55, 214-226 (2006).

${ }^{58}$ R. Das, J. P. Crutchfield, M. Mitchell, and J. E. Hanson, "Evolving globally synchronized cellular automata," in Proceedings of the Sixth International Conference on Genetic Algorithms, Pittsburgh, PA, 15-19 July 1995, edited by L. J. Eshelman (Morgan Kaufmann, San Francisco, 1995), pp. 336-343.

${ }^{59}$ W. Hordijk, "The structure of the synchonizing-CA landscape," Santa Fe Institute Technical Report No. 96-10-078 (http://www.santafe.edu/ research/publications/wplist/1996).

${ }^{60} \mathrm{M}$. Sipper, Evolution of Parallel Cellular Machines: The Cellular Programming Approach (Springer-Verlag, Heidelberg, 1997).

${ }^{61}$ H. Guclu, G. Korniss, M. A. Novotny, Z. Toroczkai, and Z. Rácz, "Synchronization landscapes in small-world-connected computer networks," Phys. Rev. E 73, 066115 (2006).

${ }^{62}$ H. Hong, B. J. Kim, M. Y. Choi, and H. Park, "Factors that predict better synchronizability on complex networks," Phys. Rev. E 69, 067105 (2004). 
${ }^{63}$ A. E. Motter, C. S. Zhou, and J. Kurths, "Enhancing complex-network synchronization," Europhys. Lett. 69, 334-340 (2005).

${ }^{64} \mathrm{~B}$. Hellwig, "A quantitative analysis of the local connectivity between pyramidal neurons in layers $2 / 3$ of the rat visual cortex," Biol. Cybern. 82, 111-121 (2000).
${ }^{65}$ S. L. Levitan, "Measuring communication structures in parallel architectures and algorithms," in The Characteristics of Parallel Algorithms, edited by L. H. Jamieson, D. B. Gannon, and R. J. Douglass (MIT Press, Cambridge, MA, 1987), pp. 101-137. 
Chaos is copyrighted by the American Institute of Physics (AIP). Redistribution of journal material is subject to the AIP online journal license and/or AIP copyright. For more information, see http://ojps.aip.org/chaos/chocr.jsp 